\title{
Enterovirus 71 structural viral protein 1 promotes autophagy by inducing an m6A-mediated PMP22 overexpression in mouse Schwann cells
}

\section{Guangming Liu}

Guangzhou Women and Children's Medical Center

\section{Danping Zhu}

Guangzhou Women and Children's Medical Center

\section{Dandan Hu}

Guangzhou Women and Children's Medical Center

\section{Suyun Li}

Guangzhou Women and Children's Medical Center

\section{Qiuyan Peng}

Guangzhou Women and Children's Medical Center

Peiqing Li ( $\square$ annie_129@126.com )

Guangzhou Medical University https://orcid.org/0000-0002-2186-1331

\section{Sida Yang}

Guangzhou Women and Children's Medical Center

\section{Research Article}

Keywords: Enterovirus 71 structural viral protein 1, m6A methylation, peripheral myelin protein 22, autophagy

Posted Date: October 26th, 2021

DOI: https://doi.org/10.21203/rs.3.rs-945661/v1

License: (9) (i) This work is licensed under a Creative Commons Attribution 4.0 International License. Read Full License 


\section{Abstract \\ Objective}

Enterovirus 71 (EV71), one of the enteroviruses responsible for the hand, foot and mouth disease (HFMD), can cause severe neurologic diseases such as brainstem encephalitis and demyelination. The molecular mechanism of demyelination is still not fully understood. This study aims to investigate the mechanism of how the EV71 structural viral protein 1, VP1 can act on host cellular pathways in mouse Schwann cells.

\section{Methods}

An EV71 VP1-expressing vector was generated and transfected into mouse Schwann cells (MSCs). Selective mRNA methylation inhibitor (DAA) was employed to identify key members of $\mathrm{m}^{6} \mathrm{~A}$ pathway that are targeted by VP1. To investigate the role of METTL14 and YTHDF1 in PMP22 expression, small interfering RNA against METTL14 and YTHDF1 was employed to knockdown the METTL14 and YTHDF1 expression in MSCs. Real-time PCR and Western blot analysis were performed to determine the expression of PMP22 and $\mathrm{m}^{6} \mathrm{~A}$ modification-associated proteins.

\section{Results}

Our results demonstrated VP1 upregulated $\mathrm{m}^{6} \mathrm{~A}$ pathway by targeting METTL14 and YTHDF1. The expression of PMP22 was decreased by inhibiting the expression of METTL14 and YTHDF1.

\section{Conclusions}

VP1 upregulates $\mathrm{m}^{6} \mathrm{~A}$ modification, which in turn causes the hypermethylation of PMP22 in MSCs, resulting in a higher level of PMP22. Ultimately, this VP1-induced PMP22 overexpression leads to MSC autophagy.

\section{Introduction}

Hand, foot and mouth disease (HFMD) is a viral infection caused by enterovirus species A and it mainly affects children under 5 years of age. HFMD is usually self-limiting, but in the cases associated with enterovirus A71 (EV-A71 or EV71), patients may develop severe or even fatal neuro-systemic complications (1). EV71 is a highly neurotropic virus that causes severe neurological symptoms such as polio-like paralysis, spinal cord myelitis, brainstem encephalitis, etc. According to a recent study, EV71 infection accounts for at least $80 \%$ of the severe cases and $90 \%$ of deaths among HFMD patients in China (2). Autopsy in EV71-related deaths revealed neurophagy (neuronal autophagy) and demyelination (3-5), which were similar to the EV71-infected mice (6-9). However, the pathogenesis of these conditions remains unclear. 
Myelin is a lipid-rich material that insulates the axons to increase the speed of the action potentials in neurons. During myelination, Schwann cells are precisely regulated to extend their membranes and wrap around axons, forming the multilayered, compact-membrane structures known as myelin sheaths (10). Myelin plays a key role in both the development of nervous system and the remyelination of damaged, demyelinated axons (10). Peripheral myelin protein 22 (PMP22) is a tetrameric transmembrane protein crucial to the development and maintenance of compact myelin, and it is expressed at high levels in the myelinating Schwann cells of the peripheral nervous system (10). A case study has reported that PMP22 is required for the normal functioning of cranial nerve motor neurons and spinal nerve sensory neurons during early development (11). Excessive accumulation of PMP22 affects protein homeostasis in cells, leading to Schwann cell dysfunction and death, as well as demyelination (12). Therefore, PMP22 needs to be tightly regulated to ensure proper myelination and therefore poses a potential therapeutic target of myelin disorders and neuron protection. Moreover, our group has previously noticed an astonishing symptomatic resemblance in patients diagnosed with PMP22 abnormality and EV71-associated neurological complications (13). We found that $80 \%$ of the EV71-associated neurological complications occurred in patients under five years of age; early symptoms include cranial nerve malfunctions such as dysphagia and impaired eye movements, suggesting a possible link to abnormal PMP22 or aberrant myelination (13). However, the mechanism of myelin damage in the extracranial cranial nerve in the early stage of EV71 infection has not been reported. In the early stage, our research group adopted the technical methods of Transmission Electron Microscopy (TEM), Quantitative real-time PCR (qPCR), Immunofluorescence, Western blot and autophagy marker showed that PMP22 expression and autophagy were enhanced in Mouse Schwann cells with VP1 overexpression, Inhibition of PMP22 expression eliminated autophagy enhancement, suggesting that PMP22 plays an important role in autophagy (14). Excessive accumulation of PMP22 affects the protein homeostasis in cells and leads to Schwann cell dysfunction and demyelination $(15,16)$.

Since PMP22 seems to be one of the primary targets of EV71, it is important to understand the mechanism by which PMP22 can be affected. Studies have shown that PMP22 undergoes methylation on the RNA level and a hypermethylation can lead to the overexpression of $\operatorname{PMP} 22(17,18)$. N6-methyladenosine $\left(\mathrm{m}^{6} \mathrm{~A}\right)$ is one of the most common post-transcriptional RNA modifications in eukaryotic cells. $\mathrm{M}^{6} \mathrm{~A}$ mainly involves three classes of proteins: 1) methylases, such as METTL3/14 and m6A-METTL-associated complex (MACOM), 2) Demethylases, such as FTO and ALKBH5, and 3) M6A methylation recognition protein, such as $Y T H(19,20)$. While $m^{6} A$ assists mRNA processing and transport, it has also been associated with the development of tumors and neurological disorders (21). Studies have also associated EV71 infection with RNA methylation and reported that the $\mathrm{m}^{6} \mathrm{~A}$ modification complex modulates the replication of EV71 (22). However, it is not clear whether EV71 infection can alter the expression of key genes during $\mathrm{m}^{6} \mathrm{~A}$ reversely. We hypothesized that EV71 infection could induce the hypermethylation of PMP22 in mouse Schwann cells (MSCs), increasing its gene dosage and resulting in the subsequent manipulation of downstream host cellular pathways.

The aim of this study was to investigate the pathogenesis of neurological complications associated to EV71 infection. We were especially interested in the development of neurophagy and demyelination in the 
fatal cases of EV71 infection. We speculated that the protein of our interest, the host peripheral myelin protein PMP22 was tightly linked to the autopsy-confirmed disease phenotypes. The overarching goal of this study was to uncover the correlations between EV71 infection, $\mathrm{m}^{6} \mathrm{~A}$ methylation and PMP22 gene regulation. By understanding the mechanisms behind these molecular processes and the host-pathogen protein interactions in vitro, we hope that our study will aid in the development of a more effective HFMD treatment that can lower the risks of EV71-associated neurological complications and deaths.

\section{Materials And Methods}

\section{Sampling and EV71 isolation}

EV71 was isolated from clinical specimens including throat and anal swabs and stools of a patient with HFMD due to EV71 infection, provided by the Center for Disease Control and Prevention of Guangdong Province (Guangzhou, China). The patient was diagnosed at Zhongshan School of Medicine, Sun Yat-sen University (Guangzhou, China). The study was approved by the ethics committee of the Guangzhou Medical University, Guangzhou Women and Children's Medical Center, IRB, Wuhan Institute of Virology, CAS (no. 2017122501, 2017 - 396 and WIVA07201904). All procedures performed involving human participants were in accordance with the ethical standards of the institutional and/or national research committee and with the 1964 Helsinki declaration and its later amendments or comparable ethical standards. Written informed consent was obtained from the legal guardians of the patient from whom EV71 was isolated for research purposes.

\section{Cell line and culture}

Mouse Schwann cells(ScienCell Research Laboratories, Inc., Cat. \#M1700)were cultured in Schwann Cell

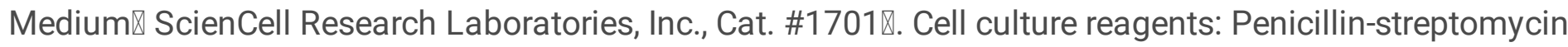
solution (Hyclone Laboratories, Inc., Cat. \#SH30010), PBS Potassium phosphate buffer (Hyclone Laboratories, Inc., Cat. \#SH30256.01B). Cell resuscitation and cell subculture were carried out according to the experimental procedure, and the cell concentration was adjusted to $5 \times 10^{7} / \mathrm{ml}$ for the experiment. Schwann cell medium containing penicillin $(100 \mathrm{U} / \mathrm{ml})$ and streptomycin $(100 \mu \mathrm{g} / \mathrm{ml})$ in poly-L-lysinecoated $(2 \mathrm{\mu g} / \mathrm{cm} 2)$ flasks at $37^{\circ} \mathrm{C}$ in a humidified atmosphere of $5 \% \mathrm{CO}_{2}$. DMEM medium was replaced every other day. The third-generation chondrocytes were counted and used to prepare cell suspension. Trypan blue staining to detect cell viability was $90 \%$, and the cell concentration was adjusted to $5 \times 10^{7} / \mathrm{ml}$ for experiments.

\section{Plasmid preparation and transfection for overexpression}

Total RNA (EV71) was extracted using TRIzol (Thermo Fisher Scientific, Inc., Cat. \#15596026) and reverse transcribed to obtain cDNA (Promega corporation, Cat. \#A3800). The 894-bp VP1 cDNA was synthesized by reverse transcription-polymerase chain reaction (RT-PCR; Takara Bio, Inc., Cat. \#2011A) using the primers: Forward 5'-ccGcTcGAGGccAccATGGGTGATGGAATTGcAGAcATGA-3' and reverse 5'-cGcGGATccTAG TGTTGTTATTTT GTcccTAcT TGT Gc-3' (Genewiz, Inc.). The PCR products were subcloned into the pEGFPc3 expression vector (Clontech Laboratories, Inc.) following manufacturer's protocol. To determine if the 
VP1 cDNA was successfully cloned into the pEGFP-c3 vector, plasmids from transformed bacteria were prepared and digested with BamHI and $X$ hol. Sequencing was performed by Sangon Biotech for confirmation of successful cloning. The results were compared with the VP1 cDNA sequence reported in the GenBank database (GenBank accession number: U55763). The EV71-VP1 overexpression plasmid is annotated as pEGFP-c3 + EV71-VP1. Cells were transiently transfected with $2 \mathrm{mg}$ plasmid using Lipofectamine 2000 (Invitrogen; Thermo Fisher Scientific, Inc.) for $24 \mathrm{~h}$, according to the manufacturer's instructions.

\section{Gene silencing by siRNA}

Genes of interest are targeted and silenced by siRNA. METTL14 and YTHDF1 siRNA (minic) are ordered from Santa Cruz Biotechnology., Inc. (Cat. \#sc-42037, Santa Cruz, CA, USA) (Table 1, Fig. 3A). siRNA was transfected into mouse Schwann cells by Lipofectamine 2000 ( Invitrogen,Cat. \#11668019) and Opti-MEM (Invitrogen־Cat. \#31985070). Non-targeting siRNA sequences were transfected into the cells as negative controls.

Table 1

Target sequence for siRNA-METTL14 and siRNA-YTHDF1

\begin{tabular}{|c|c|c|c|c|}
\hline & Ranking & siRNA ID & Sequence Start & Target sequence \\
\hline \multirow[t]{3}{*}{ siRNA-METTL14 } & 1 & SASI_Mm01_00071493 & 555 & agaatttcatcagggatgtag \\
\hline & 2 & SASI_Mm01_00071495 & 761 & agagagactggcatcactgcg \\
\hline & 3 & SASI_Mm02_00351698 & 1399 & tcccagaggtggaggaagggg \\
\hline \multirow[t]{3}{*}{ siRNA-YTHDF1 } & 1 & SASI_Mm01_00078555 & 407 & ccaccatccattggatttcct \\
\hline & 2 & SASI_Mm01_00078556 & 1726 & acctgtcacaaactcccgtga \\
\hline & 3 & SASI_Mm01_00078557 & 1264 & tgacagtaactctgttggaaa \\
\hline \multicolumn{5}{|c|}{ Note: METTL14 methyltransferase like 14 [ Mus musculus (house mouse)]. } \\
\hline \multicolumn{5}{|c|}{ Gene ID: 210529, updated on 8-Nov-2020. } \\
\hline \multicolumn{5}{|c|}{ Mus musculus methyltransferase like 14 (Mett|14), mRNA. } \\
\hline \multicolumn{5}{|c|}{ NCBI Reference Sequence: NM_201638.2 } \\
\hline \multicolumn{5}{|c|}{ Ythdf1 YTH N6-methyladenosine RNA binding protein 1 [ Mus musculus (house mouse)]. } \\
\hline \multicolumn{5}{|c|}{ Gene ID: 228994, updated on $20-0 c t-2020}$. \\
\hline \multicolumn{5}{|c|}{ Mus musculus YTH N6-methyladenosine RNA binding protein 1 (Ythdf1), mRNA. } \\
\hline NCBI Reference $S$ & quence: $\mathrm{N}$ & 173761.3. & & \\
\hline
\end{tabular}

\section{Experimental grouping and operation}


$\mathrm{m}^{6} \mathrm{~A}$-related gene detection experiment

Mouse Schwann cells were divided into three groups: cell (normal control group), NC (negative control group), SC + EV71-VP1 (experimental group). Gene expression was quantified by qPCR at 24-hour time point and protein expression was confirmed by immunoblotting at 48-hour respectively (Please see methods below).

\section{DAA treatment of mouse Schwann cells}

Selective mRNA methylation inhibitor 3-deazaadenosine (DAA) (Selleck Chemicals, Cat. \# S0787, Huston, TX, USA) was employed to determine which elements involved in the $\mathrm{m}^{6} \mathrm{~A}$ pathway are targeted by VP1. Mouse Schwann cells were treated with DAA [3.9 $\mu \mathrm{M}(\mathrm{Ki}), 60 \mathrm{mg} / \mathrm{ml}]$.

mRNA methylation

Mouse Schwann cells were divided into four groups: cell (normal control group), NC (negative control group), EV71-VP1 (experimental control group) and EV71-VP1-siRNA-METTL14 or-YTHDF1 (siRNA experimental group).

\section{Quantitative real-time PCR (qPCR)}

The total RNA of the sample was extracted using TRE-Trizol (Invitrogen,Cat. \#15596-026). The design of fluorescence quantitative PCR primers is shown in Table 2. Complementary DNA (CDNA) was obtained by the reverse transcription of mRNA, which was performed in strict accordance with the instructions of TaKaRa PrimeScript II 1st Strand cDNA Synthesis Kit (TaKaRa Bio Inc., Cat. \# D6210A). The preparation and reaction parameters of the fluorescent quantitative PCR reaction System were performed according to the instructions of the TaKaRa SYBR® Premix Ex Taq ${ }^{\text {TM }}$ II (Perfect Real Time) kit (TaKaRa Bio Inc., Cat. \# RR039B). Amplification and fusion curves of Real Time PCR were performed using ABI PRISM® 7500 Sequence Detection System (REAL-TIME PCR System). Fluorescence quantitative RT-PCR data was analysis using the SDSShell Software1.6 software, and the $2^{-\Delta \Delta}$ Ct method to calculate. 
Table 2

Primers used for fluorescence quantitative PCR

\begin{tabular}{|lllll|}
\hline Gene & ID & Forward primer & Reverse primer & $\begin{array}{c}\text { Product } \\
\text { length } \\
\text { (bp) }\end{array}$ \\
\hline ALKBH5 & 268420 & ATTGCCACCCAGCTATGCTT & CTCATCTTCACCTTGCGGGT & 273 \\
\hline EV71-VP1 & 1461111 & TCGTGCGCTGACATCCTTAC & ATGGTTCAACACACACCGGG & 166 \\
\hline FTO & 26383 & TGAAAGTGAGGACGAGTCCAG & TGAGTGGAACTAAACCGAGGC & 201 \\
\hline METTL14 & 210529 & CTGGCATCACTGCGAATGAG & CGCAAGCATACTCTCCCAAG & 142 \\
\hline METTL3 & 56335 & ATCTTGGCTCTATCCGGCTG & CCTTAAATCCAAGTGCCCAGAG & 231 \\
\hline YTHDC1 & 231386 & GGAAGAGAACCAGAGCACGG & CCATCTTTCTCCTCCCGGC & 219 \\
\hline YTHDF1 & 228994 & CGTGATACACAGGAGGTGCC & CGATTCTGTCTTTCCTTACGCAC & 152 \\
\hline YTHDF2 & 213541 & CAGGCAAGGCCGAATAATGC & TCTCCGTTGCTCAGTTGTCC & 167 \\
\hline YTHDF3 & 229096 & TCAGAGACCTAAAGGGCAAGG & TGGCATAGGCTGATCTCCAG & 235 \\
\hline Rn18s & 19791 & CCTGGATACCGCAGCTAGGA & GCGGCGCAATACGAATGCCCC & 112 \\
\hline PMP22* & & cTGcCAGcTcTTCAcTcTcA & GTTGAcATGcCAcTCAcTGT & \\
\hline GAPdH & & GGccTccAAGGAGTAAGAAA & GcccCTcCTGTTATTATGG & \\
\hline Note: * PMP22, peripheral myelin protein 22. & & \\
\hline
\end{tabular}

\section{Western blot}

Mouse Schwann cells were lysed with RIPA lysis and extraction buffer (Thermo Fisher Scientific, Inc, Cat. \#89900). Protein concentration was determined by the bicinchoninic acid protein assay kit (Beyotime Institute of Biotechnology, Cat. \# P0012). Then, $50 \mathrm{ng}$ of each protein sample was separated by $10 \%$ SDSPAGE and transferred to polyvinylidene fluoride membranes. The membranes were blocked with $5 \%$ nonfat milk powder in Tris-buffered saline/Tween 20 (TBST) at room temperature and then incubated with antiGAPdH (1:1 000, Abcam, Cat. \# ab181602) for 1-2 h at room temperature, and then with peroxidaseconjugated secondary antibody (1:4 000, Thermo Fisher Scientific, Inc., Cat. \# 31460) for $1 \mathrm{~h}$ at room temperature. Membranes were visualized with an enhanced chemiluminescent development reagent (BeyoECL Plus, GE Healthcare, Cat. \# 28935599) and densitometry analysis was performed using Quantity One (Bio-Rad Laboratories, Inc.).

Primary antibodies used in the study were: Enterovirus 71 VP1 Monoclonal Antibody (3656), Thermo Fisher, Cat. \# MA5-33257; Rabbit Anti-METTL14 antibody \& Rabbit Anti-FTO antibody \& Rabbit Anti-ALKBH5 antibody, Bioss, Cat. \# bs-17608R, bs-7056R, bs-20540R; Recombinant Anti-METTL3 antibody [EPR18810] \& Recombinant Anti-YTHDF1 antibody [EPR22349-16] \& Recombinant Anti-YTHDF2 antibody [EPR20318] \& Recombinant Anti-YTHDF3 antibody [EPR21912-3] \& Recombinant Anti-YTHDC1 antibody [EPR21821], abcam, Cat. \#ab195352 \& ab252346 \& ab220163 \& ab220161\& ab220159. Secondary antibodies are 
Rabbit Anti-Mouse IgG (H + L), 1:4 000, Southern Biotech, Cat. \# 6170-05; Goat Anti-Rabbit lgG (H + L chain specific), 1:5 000, Southern Biotech, Cat. \#4050-05).

\section{Statistical analysis}

All experiments were repeated at least three times. data are expressed as mean \pm standard error (SE). Statistical significance was assessed by the Student's t test or one-way ANOVA with the Least Significance Difference (LSD) post hoc test, using the SPSS 16.0 statistical software (SPSS, IBM). P $<0.05$ indicates statistical significance.

\section{Results}

Key elements involved in $\mathrm{m}^{6} \mathrm{~A}$ are upregulated on both RNA and protein levels by VP1

As mentioned, PMP22 undergoes post-transcriptional methylation $(17,23)$. Previous studies have shown that hypermethylation results in higher level of PMP22 $(17,18)$, which may increase the risk of tumorigenesis and nervous system disease (21). Therefore, to understand the pathogenesis of EV71 infection, it is important to explore whether VP1 overexpression would lead to hypermethylation. To achieve this goal, we examined the expression of key genes involved in $\mathrm{m}^{6} \mathrm{~A}$ modification in VP1-transfected mouse Schwann cells. qRT-PCR results showed that the gene expression levels of METTL3/14 and YTHDF1/2/3 were significantly increased. (Supplementary Table 1, Fig. 1)

We also examined the changes in proteins involved in methylation by harvesting Schwann cells 48 hours after transfection with EV71-VP1. Our proteins of interest included METTL3, METTL14, YTHDF1, YTHDF2, YTHDF3, YTHDC1, FTO and ALKBH5. The results showed that the levels of most proteins, specifically METTL3, METTL14, YTHDF1, YTHDF2 and YTHDF3 increased significantly in the presence of VP1, while FTO decreased significantly. (Supplementary Table 2, Fig. 2)

The suppression of METTL14 or YTHDF1 downregulates the expression of PMP22

To identify the proteins that are actively involved in $\mathrm{m}^{6} \mathrm{~A}$ modification, EV71-VP1-overexpressing mouse Schwann cells were treated with an mRNA methylation inhibitor DAA (Fig. 3) (24). RT-PCR results showed that the gene expression of METTL 3/14 and YTHDF 1/2 decreased significantly after DAA treatment, even when VP1 is overexpressed (Supplementary Table 3, Fig. 3). These results further verified that METTL 3/14 and YTHDF 1/2 were key members of the methylation pathway that are affected by VP1.

Next, we focused on the two key elements of $\mathrm{m}^{6} \mathrm{~A}$ to see whether inactivation of METTL14 or YTHDF1 alone could rescue the increase in PMP22 mediated by VP1. In the presence of VP1, the level of PMP22 increased in comparison to un-transfected and negative controls (Fig. 4). When expressions of METTL14 and YTHDF1 were suppressed by the targeting siRNA respectively, the level of PMP22 decreased, counteracting the effect of VP1(Fig. 4).

\section{Discussion}


The interactions between host and viral proteins are crucial to the establishment of a viral infection. For instance, previous studies have found that through interacting with VP1, the human scavenger receptor class B member 2 (SCARB2) serves as a cellular receptor and mediates the endocytosis of EV71, allowing viral entry (25). Our study investigated beyond viral adsorption and delved further into the pathogenesis of EV71-associated neurological complications by unravelling any interaction between viral proteins and downstream host pathways. Specifically, we speculated that the host peripheral myelin protein PMP22 might be a potential target of EV71 during infection, suggested by a positive correlation between the EV71 structural protein VP1 and PMP22 in mouse Schwann cells. Furthermore, our study has provided a possible mechanism responsible for the interaction between VP1 and PMP22. Our results have demonstrated that VP1 is able to drive up the expression of key elements in $\mathrm{m}^{6} \mathrm{~A}$ RNA modification, thus upregulating the methylation of PMP22 and subsequently its expression in mouse Schwann cells. Subsequently, high levels of PMP22 promote autophagy in mouse Schwann cells, leading to Schwann cell dysfunction and death, disabling them from producing intact myelin sheaths. Demyelination impairs signal propagation along the axons and causes axons to deteriorate, leading to neurological problems such as neuronal necrosis and neuronophagia (26). Therefore, our proposed mechanism behind virus-induced demyelination of peripheral nerves may provide more insights in developing effective early interventions to the neurological complications among HFMD patients.

EV71 carries four structural proteins: VP1, VP2, VP3, and VP4. VP1 homodimers are the main component of the icosahedral viral capsid, which contributes to the pathogenicity and stability of EV71 and allows it to survive in the gastrointestinal tract $(27,28)$. Our previous study found that VP1 overexpression induced autophagy in Schwann cells in mice. The autophagy pathway is most likely to be activated through the interaction between VP1 and a host protein, PMP22. Peripheral myelin protein PMP22 is an essential player in regulation of the autophagy pathway in Schwann cells. When PMP22 expression was suppressed by a targeting small interfering RNA, autophagy was downregulated, and mouse Schwann cells can maintain their integrity $(14,29)$. The adverse effect of PMP22 accumulation has been described in other studies, where excessive PMP22 affects protein homeostasis in cells (12). However, whether autophagy plays a role in the pathogenesis of neurological complication remains a question. Autophagy plays dual roles in the nervous system. Excessive autophagy may be protective in chronic neurodegenerative diseases but detrimental in acute neural damages (30). EV71 can cause neuronal autophagy $(4,31)$, while autophagy inhibition intervention can reduce viral infections, neurological symptoms and reduce mortality (32). Nevertheless, autophagy and PMP22 may still pose as interesting therapeutic targets, as their regulations could be tightly linked to the progression of EV71 infection. Further studies are needed to fully understand the effect of autophagy on EV71 infection as well as how it might be fine-tuned.

As PMP22 and autophagy may be closely related to the neurological disease phenotype during complications after EV71 infection, we were curious about the driving force of the PMP22 upregulation. Studies have also shown that the expression of PMP22 is sensitive to gene doses; a slight increase in the methylation of PMP22 on the RNA level causes PMP22 to overexpress $(17,18)$. N6-methyladenosine $\left(\mathrm{m}^{6} \mathrm{~A}\right)$ modification has been associated to myelination, as well as EV71 replication in the host (22). Specifically, $\mathrm{m}^{6} \mathrm{~A}$ is involved in chromatin modification and the epigenetic controls of myelin sheath regeneration and 
axonal repair $(33,34)$. During EV71 infection, $\mathrm{m}^{6} \mathrm{~A}$ modification complex modulates viral replication through an interaction between its key component METTL3 and a viral RNA polymerase (22). In the present study, we have shown that viral structural protein VP1 is also involved in m[anipulating $\mathrm{m}^{6} \mathrm{~A}$ modifications. We have also identified two host proteins targeted by VP1, METTL14 and YTHDF1, key members of the $\mathrm{m}^{6} \mathrm{~A}$ pathway, and we have shown that VP1 overexpression could lead to the hypermethylation of PMP22. From our results, we are able to propose a possible mechanism of how PMP22 is upregulated during EV71 infection. Further experiments are needed to illustrate a more detailed mechanism of VP1 targeting on $\mathrm{m}^{6} \mathrm{~A}$.

Considering the role of VP1 in manipulating autophagy, $\mathrm{m}^{6} \mathrm{~A}$ methylation and PMP22 expression, we conclude that VP1 may be a potential therapeutic target for EV71-induced demyelinating damage. However, as VP1 has been shown to have other important functions in the viral life cycle, such as in the adsorption of the viral particle, it is likely that the VP1 also interferes with other host cellular pathways. In summary, our study has not only uncovered a potential therapeutic target specifically for EV71 infections, but it has also improved our understanding of host-pathogen interactions in general. In the long run, we hope that the information that we are getting from this study could aid in developing better treatments for the early intervention of EV71-related neurological diseases.

\section{Abbreviations}

ALKBH5, AlkB homolog 5;

ER, endoplasmic reticulum

EV71, enterovirus 71

FTO, fat mass and obesity-associated protein

$m^{6} \mathrm{~A}$, N6-methyladenosine

METTL14, methyltransferase-like protein 14;

METTL3, methyltransferase-like protein 3

PMP22, peripheral myelin protein 22

qPCR, quantitative real-time PCR

SC, Schwann cells

VP1, viral protein 1

YTHDC1, YTH domain containing protein 1

YTHDF1, YTH N(6)-Methyladenosine RNA Binding Protein 1 
YTHDF2, YTH N(6)-Methyladenosine RNA Binding Protein 2

YTHDF3, YTH N(6)-Methyladenosine RNA Binding Protein 3

\section{Declarations}

\section{Ethics approval and consent to participate}

The study was approved by the ethics committee of the Guangzhou Medical University, Guangzhou Women and Children's Medical Center, IRB, Wuhan Institute of Virology, CAS (no. 2017122501, 2017-396 and WIVA07201904)

\section{Consent for publication}

Not applicable.

\section{Availability of data and materials}

The datasets used and/or analyzed during the current study are available from

the corresponding author on reasonable request.

\section{Competing interests}

The authors declare that they have no competing interest

\section{Funding}

This work was supported by the National Natural Science Foundation of China (No. 81801206), Guangdong Natural Science Foundation (No.2020A1515010014), Science and Technology Key Project for People's Livelihood of Guangzhou, China (No. 201803010026) and the Innovative Project of Children's Research Institute, Guangzhou Women and Children's Medical Center, China (No. Pre-NSFC-2019-002, NKE-PRE-2019015 and Pre-NSFC-2019-007). The funders had no role in study design, data collection, and analysis, decision to publish, or preparation of the manuscript.

\section{Authors' contributions}

Guangming Liu wrote the manuscript. Danping Zhu performed experiments. Suyun Li performed specimen detection. Dandan Hu supervised quality of experiments. Peiqing Li conceived the study, revised and reviewed the manuscript. Sida Yang supervised quality of Clinical and laboratory data. All authors reviewed the results and approved the final version of the manuscript.

\section{Acknowledgements}

Not applicable 


\section{References}

1. Cox B, Levent F (2018) Hand, Foot, and Mouth Disease. Jama 320(23):2492

2. Xing W, Liao Q, Viboud C, Zhang J, Sun J, Wu JT, Chang Z, Liu F, Fang VJ, Zheng Y et al (2014) Hand, foot, and mouth disease in China, 2008-12: an epidemiological study. The Lancet Infectious diseases 14(4):308-318

3. Jiang M, Wei D, Ou WL, Li KX, Luo DZ, Li YQ, Chen E, Nong GM (2012) Autopsy findings in children with hand, foot, and mouth disease. N Engl J Med 367(1):91-92

4. Yang SD, Li PQ, Huang YG, Li W, Ma LZ, Wu L, Wang N, Lu JM, Chen WQ, Liu GM et al (2018) Factors associated with fatal outcome of children with enterovirus A71 infection: a case series. Epidemiol Infect 146(6):788-798

5. Li M, Kong XP, Liu H, Cheng LX, Huang JL, Quan L, Wu FY, Hao B, Liu C, Luo B (2015) [Expression of EV71-VP1, PSGL-1 and SCARB2 in Tissues of Infants with Brain Stem Encephalitis]. Fa yi xue za zhi 31(2):97-101, 104

6. Jin Y, Sun T, Zhou G, Li D, Chen S, Zhang W, Li X, Zhang R, Yang H, Duan G. Pathogenesis Study of Enterovirus 71 Using a Novel Human SCARB2 Knock-In Mouse Model. mSphere. 2021;6(2)

7. Zhang H, Song Z, Zou J, Feng Y, Zhang J, Ren L, Zhang X, Hu Y, Yuan Z, Yi Z (2020) An infectious clone of enterovirus 71(EV71) that is capable of infecting neonatal immune competent mice without adaptive mutations. Emerging microbes infections 9(1):427-438

8. Zhu L, Hao X, Cao J, Xie X, Wang H (2020) BNIP3 deletion ameliorated enterovirus 71 infection-induced hand, foot and mouth disease via inhibiting apoptosis, autophagy, and inflammation in mice. Int Immunopharmacol 87:106799

9. Yu P, Bao L, Xu L, Li F, Lyu Q, Yao Y, Qin C (2014) [Histopathological changes in EV71-infected mouse model:a transmission electron microscopic study]. Zhonghua Bing Li Xue Za Zhi 43(2):109-113

10. Li J, Parker B, Martyn C, Natarajan C, Guo J (2013) The PMP22 gene and its related diseases. Mol Neurobiol 47(2):673-698

11. Saporta MA, Katona I, Zhang X, Roper HP, McClelland L, Macdonald F, Brueton L, Blake J, Suter U, Reilly MM et al (2011) Neuropathy in a human without the PMP22 gene. Arch Neurol 68(6):814-821

12. Watila MM, Balarabe SA (2015) Molecular and clinical features of inherited neuropathies due to PMP22 duplication. Journal of the neurological sciences 355(1-2):18-24

13. Yang SD, Li PQ, Li YM, Li W, Lai WY, Zhu CP, Tao JP, Deng L, Liu HS, Ma WC et al (2017) Clinical manifestations of severe enterovirus 71 infection and early assessment in a Southern China population. BMC Infect Dis 17(1):153

14. Li P, Yang S, Hu D, Wei D, Lu J, Zheng H, Nie S, Liu G, Yang H (2019) Enterovirus 71 VP1 promotes mouse Schwann cell autophagy via ER stress-mediated PMP22 upregulation. Int J Mol Med 44(2):759-767

15. Marinko JT, Carter BD, Sanders CR (2020) Direct relationship between increased expression and mistrafficking of the Charcot-Marie-Tooth-associated protein PMP22. J Biol Chem 295(34):1196311970

Page 12/18 
16. Stavrou M, Sargiannidou I, Christofi T, Kleopa KA (2021) Genetic mechanisms of peripheral nerve disease. Neurosci Lett 742:135357

17. Huehne K, Rautenstrauss B (2001) Transcriptional startpoints and methylation patterns in the PMP22 promoters of peripheral nerve, leukocytes and tumor cell lines. Int J Mol Med 7(6):669-675

18. Ma KH, Hung HA, Srinivasan R, Xie H, Orkin SH, Svaren J (2015) Regulation of Peripheral Nerve Myelin Maintenance by Gene Repression through Polycomb Repressive Complex 2. The Journal of neuroscience: the official journal of the Society for Neuroscience 35(22):8640-8652

19. Chen M, Wong CM (2020) The emerging roles of N6-methyladenosine (m6A) deregulation in liver carcinogenesis. Mol Cancer 19(1):44

20. Lee Y, Choe J, Park OH, Kim YK (2020) Molecular Mechanisms Driving mRNA Degradation by m(6)A Modification. Trends Genet 36(3):177-188

21. Chen X, Sun YZ, Liu H, Zhang L, Li JQ, Meng J (2019) RNA methylation and diseases: experimental results, databases, Web servers and computational models. Brief Bioinform 20(3):896-917

22. Hao H, Hao S, Chen H, Chen Z, Zhang Y, Wang J, Wang H, Zhang B, Qiu J, Deng F et al (2019) N6methyladenosine modification and METTL3 modulate enterovirus 71 replication. Nucleic acids research 47(1):362-374

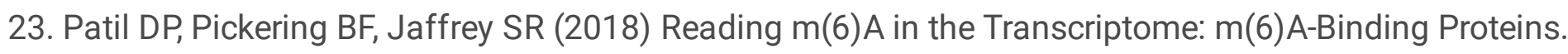
Trends in cell biology 28(2):113-127

24. Bader JP, Brown NR, Chiang PK, Cantoni GL (1978) 3-Deazaadenosine, an inhibitor of adenosylhomocysteine hydrolase, inhibits reproduction of Rous sarcoma virus and transformation of chick embryo cells. Virology 89(2):494-505

25. Zhou D, Zhao Y, Kotecha A, Fry EE, Kelly JT, Wang X, Rao Z, Rowlands DJ, Ren J, Stuart DI (2019) Unexpected mode of engagement between enterovirus 71 and its receptor SCARB2. Nature microbiology 4(3):414-419

26. Love S (2006) Demyelinating diseases. J Clin Pathol 59(11):1151-1159

27. Shi M, Zhou Y, Cao L, Ding C, Ji Y, Jiang Q, Liu X, Li X, Hou X, Peng H et al (2013) Expression of enterovirus 71 capsid protein VP1 in Escherichia coli and its clinical application. Braz J Microbiol 44(4):1215-1222

28. Plevka P, Perera R, Cardosa J, Kuhn RJ, Rossmann MG. Crystal structure of human enterovirus 71. Science (New York). 2012;336(6086):p 1274

29. Hu DD, Mai JN, He LY, Li PQ, Chen WX, Yan JJ, Zhu WD, Deng L, Wei D, Liu DH et al (2017) Glucocorticoids Prevent Enterovirus 71 Capsid Protein VP1 Induced Calreticulin Surface Exposure by Alleviating Neuronal ER Stress. Neurotox Res 31(2):204-217

30. Ginet V, Spiehlmann A, Rummel C, Rudinskiy N, Grishchuk Y, Luthi-Carter R, Clarke PG, Truttmann AC, Puyal J (2014) Involvement of autophagy in hypoxic-excitotoxic neuronal death. Autophagy 10(5):846-860

31. Lee YR, Wang PS, Wang JR, Liu HS (2014) Enterovirus 71-induced autophagy increases viral replication and pathogenesis in a suckling mouse model. Journal of biomedical science 21(1):80 
32. Owino CO, Chu JJH (2019) Recent advances on the role of host factors during non-poliovirus enteroviral infections. Journal of biomedical science 26(1):47

33. Berry KP, Lu QR (2020) Chromatin modification and epigenetic control in functional nerve regeneration. Semin Cell Dev Biol 97:74-83

34. Ma KH, Duong P, Moran JJ, Junaidi N, Svaren J (2018) Polycomb repression regulates Schwann cell proliferation and axon regeneration after nerve injury. Glia 66(11):2487-2502

\section{Figures}
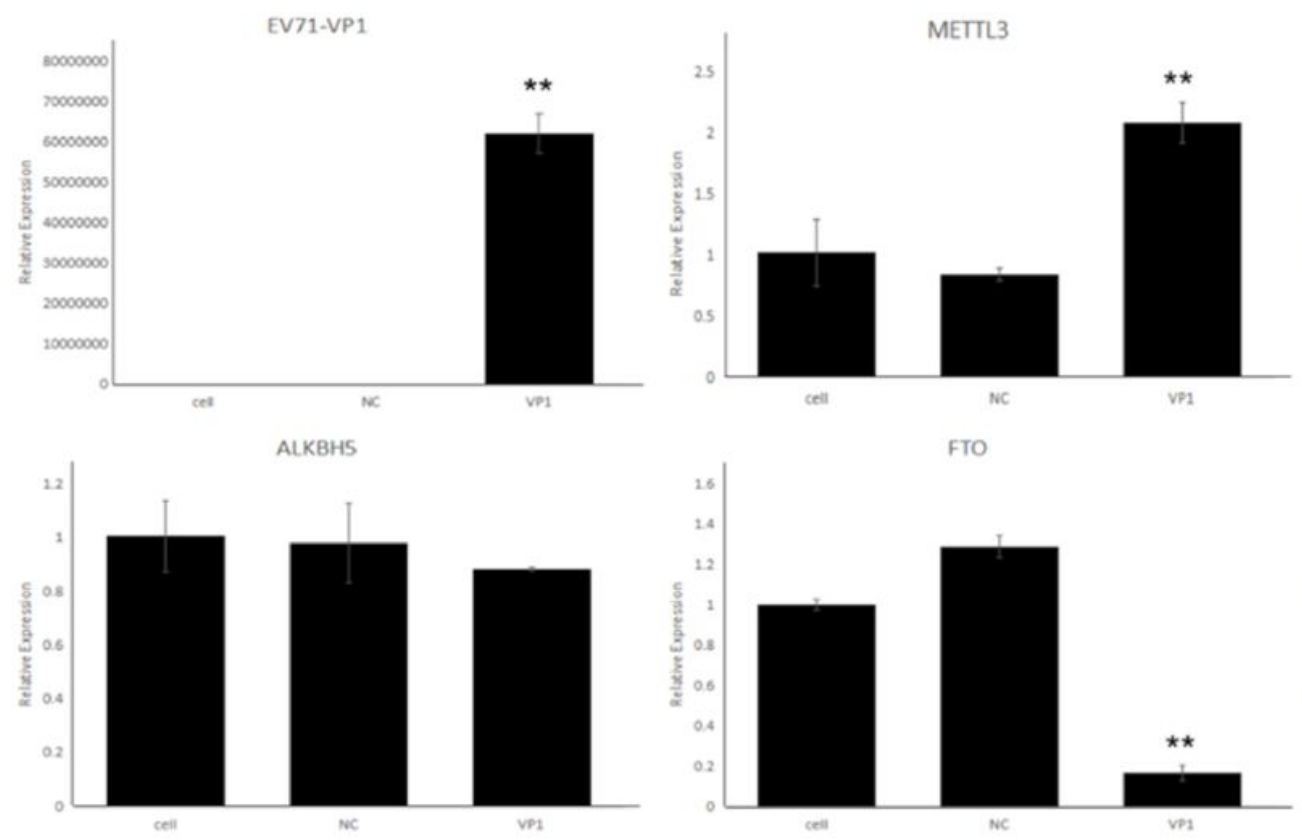

YTHDF1

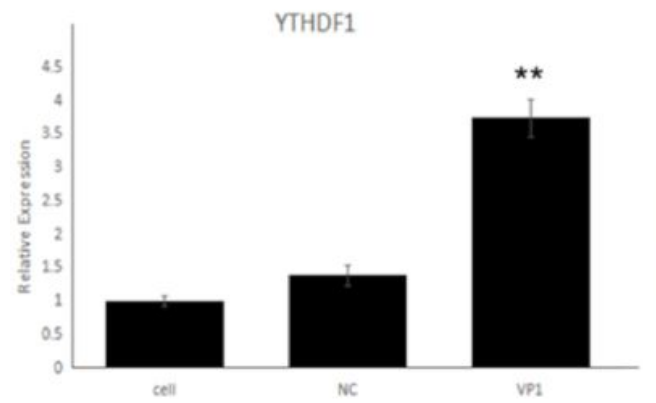

FTO

YTHDF2

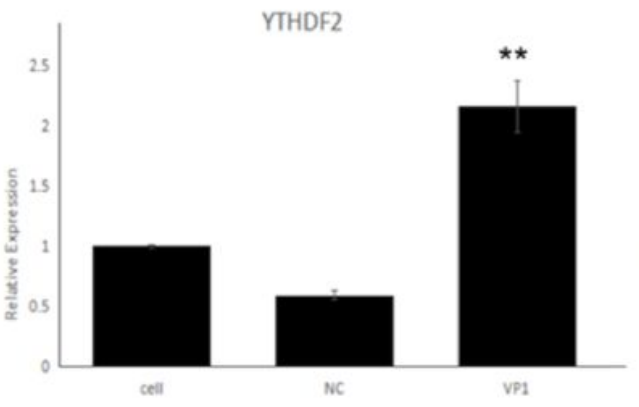

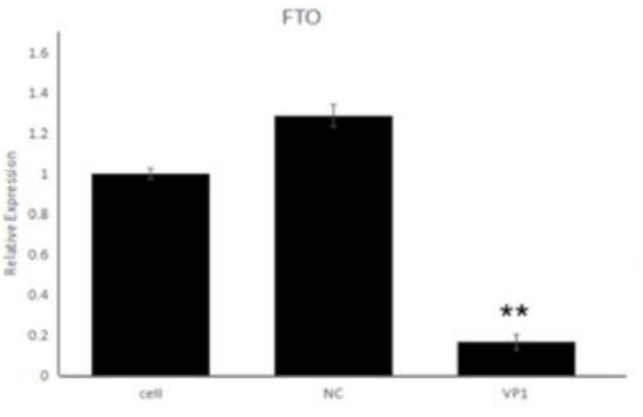

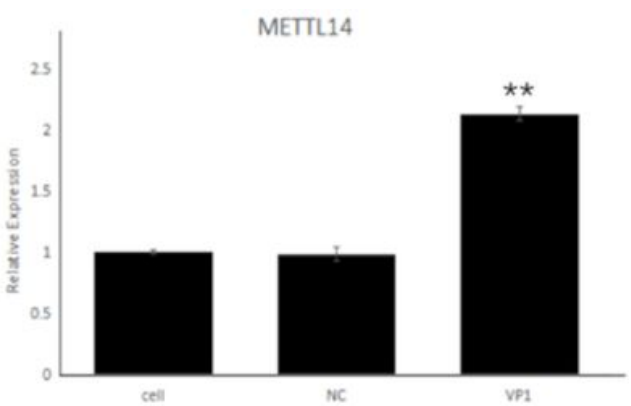

YTHDC1

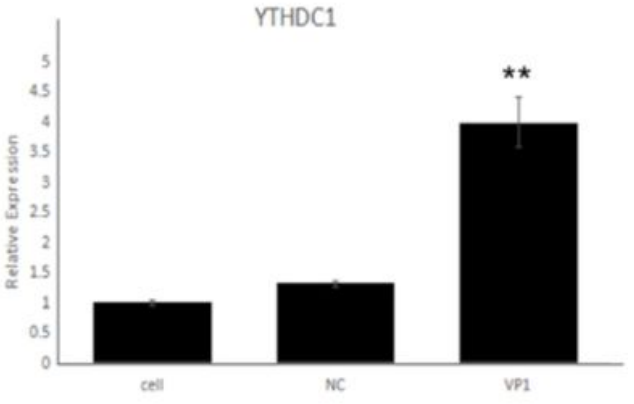

YTHDF3

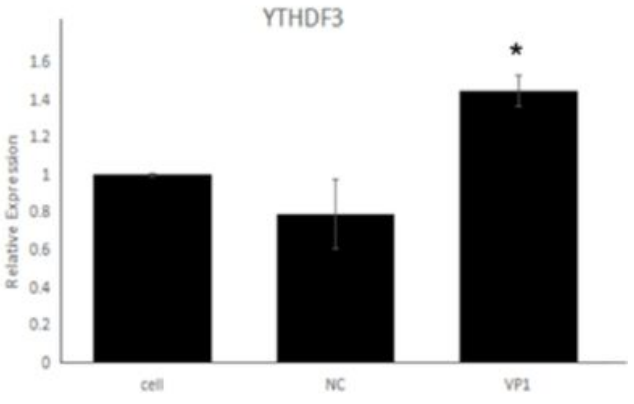

Figure 1

Transfection of EV71-VP1 affected the expression of key genes involved in m6A in mouse Schwann cells. qRT-PCR gene level was detected 24h after transfection of mouse Schwann cells with PEGFP-C3-VP1 plasmid. Un-transfected cells and PEGFP-C3-transfected cells were used as blank control and negative control, respectively. As shown in the figure. The expression levels of MRA of key methylated molecules METTLE 3/14 and YTHDF 1/2/3 were significantly increased. METTL3囚methyltransferase-like protein 3; METTL14囚methyltransferase-like protein 14; ALKBH5『AlkB homolog 5; FTO『fat mass and obesityassociated protein; YTHDC1『YTH domain containing protein 1; YTHDF1『YTH N(6)-Methyladenosine RNA 
Binding Protein 1; YTHDF2囚YTH N(6)-Methyladenosine RNA Binding Protein 2; YTHDF3囚YTH N(6)Methyladenosine RNA Binding Protein 3.
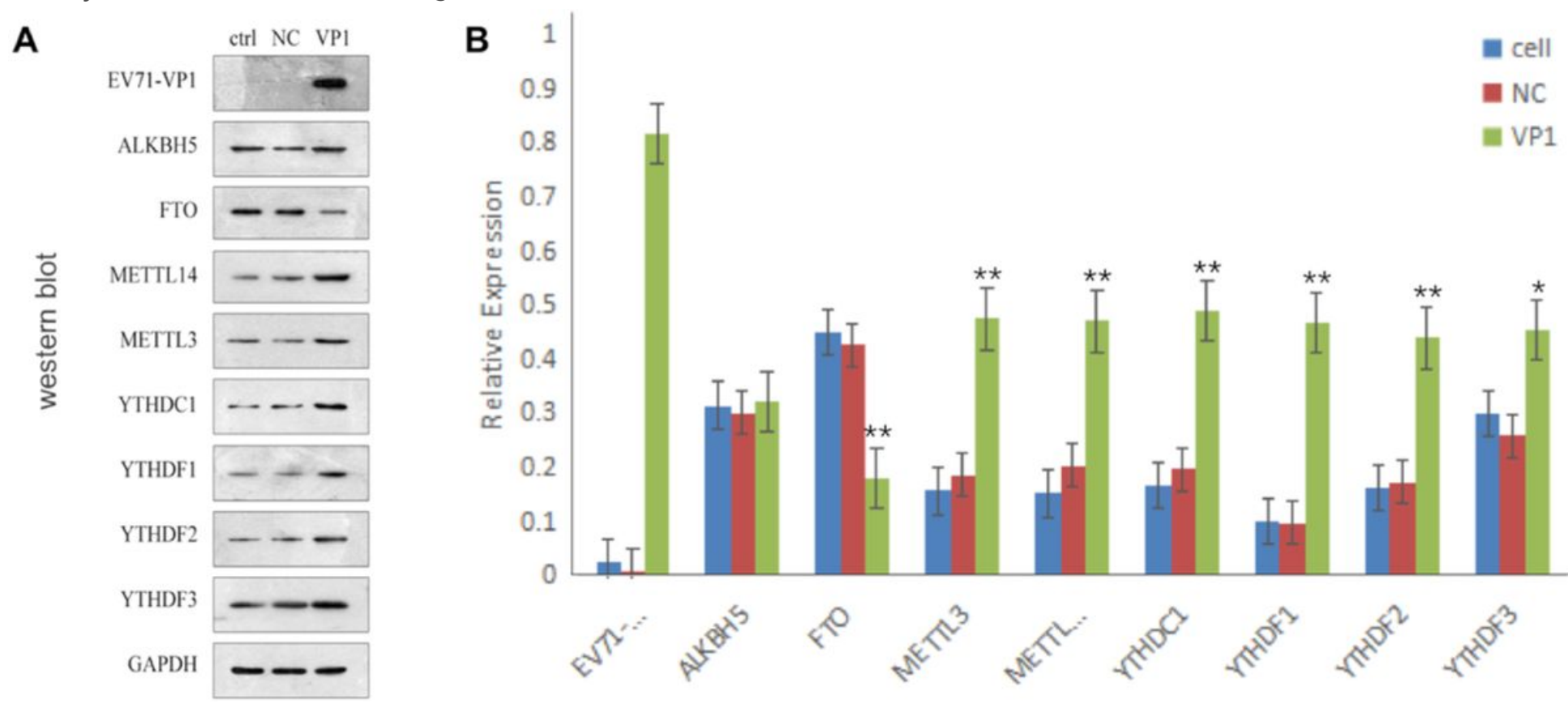

\section{Figure 2}

Western blot for m6A key proteins and their grayscale analysis in Schwann mouse cells. Proteins involved in m6A were detected by western blot 48h after transfection with VP1 plasmid. Un-transfected cells and NC cells were used as blank control and negative control, respectively. As shown in the western blot, the levels of METTL3/14 and YTHDF 1/2/3 significantly increased $48 \mathrm{~h}$ after transfection. METTL3》 methyltransferase-like protein 3; METTL14囚methyltransferase-like protein 14; ALKBH5『AlkB homolog 5; FTO $₫$ fat mass and obesity-associated protein; YTHDC1 $₫$ YTH domain containing protein 1; YTHDF1『YTH N(6)-Methyladenosine RNA Binding Protein 1; YTHDF2هYTH N(6)-Methyladenosine RNA Binding Protein 2; YTHDF3囚YTH N(6)-Methyladenosine RNA Binding Protein 3. 
A

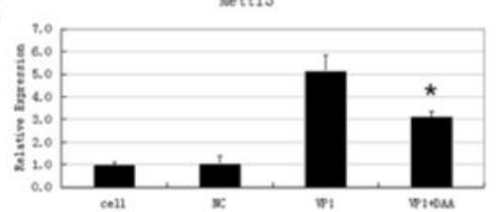

Wett114

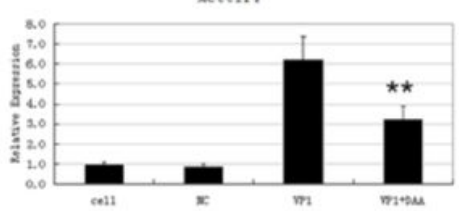

YTHDF 1

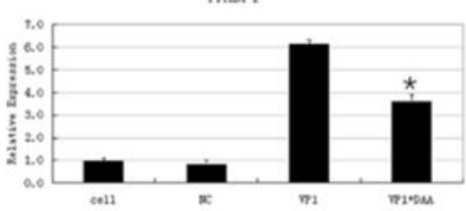

TTHDF2

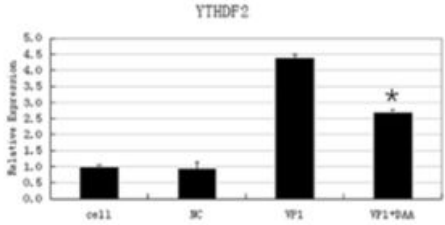

B

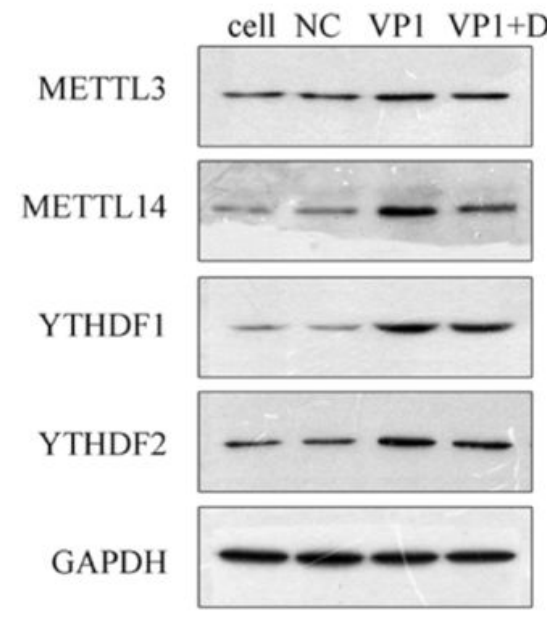

C

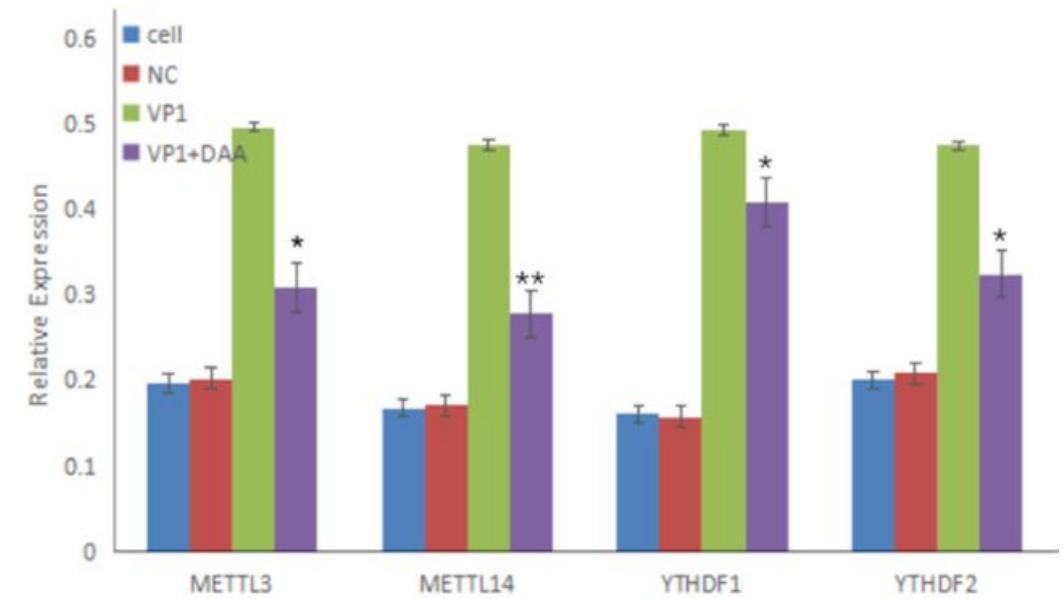

\section{Figure 3}

Transfection of VP1-DAA affected the key M6A methylated genes in mouse Schwann cells. qRT-PCR gene level was detected 24h after transfection of mouse Schwann cells with VP1-DAA. Un-transfected cells and NC cells were used as blank control and negative control, respectively. A: The expression levels of RNA of key methylated molecules METTLE 3/14 and YTHDF 1/2 were significantly downregulated. B and C: Protein levels of the key methylated molecules METTLE 3/14 and YTHDF 1/2 were significantly decreased. DAA: mRNA methylation inhibitor; METTL3囚methyltransferase-like protein 3; METTL14囚methyltransferase-like protein 14; ALKBH5囚AlkB homolog 5; FTO囚fat mass and obesity-associated protein; YTHDC1هYTH domain containing protein 1; YTHDF1囚YTH N(6)-Methyladenosine RNA Binding Protein 1; YTHDF2 $\triangle Y T H ~ N(6)-$ Methyladenosine RNA Binding Protein 2; YTHDF3囚YTH N(6)-Methyladenosine RNA Binding Protein 3. 
A

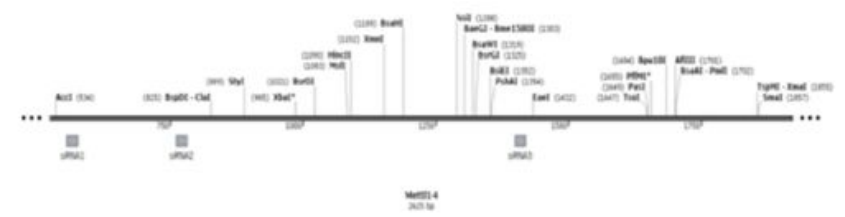

cell NC VP1 VP1+METTL14si

B
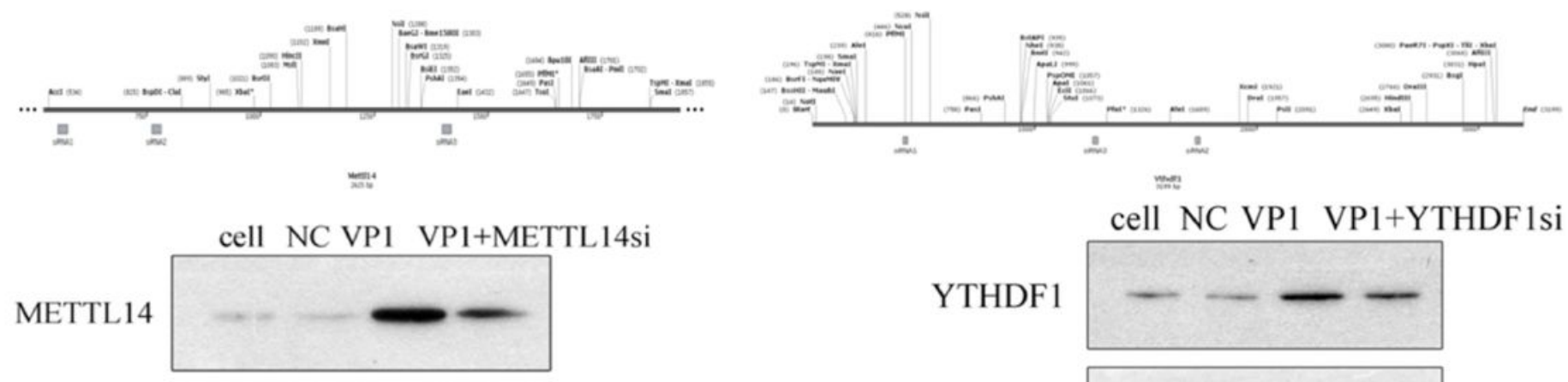

GAPDH

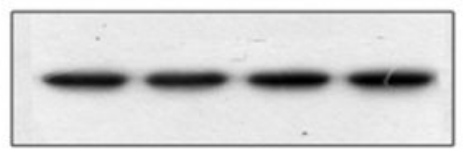

METTL14

C

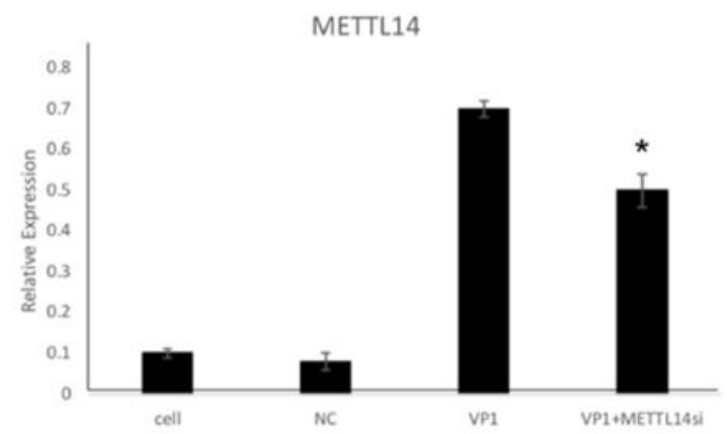

cell NC VP1 VP1+METTL14si

D

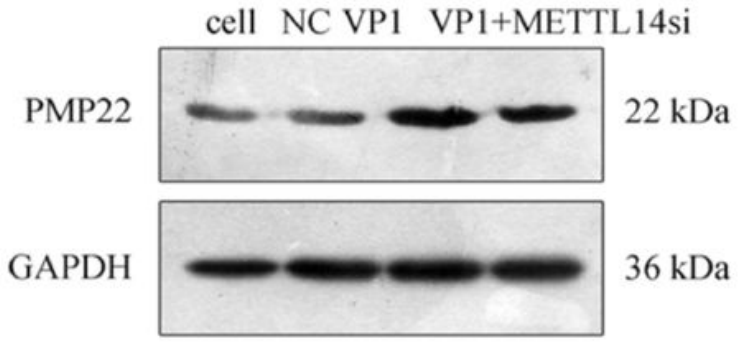

PMP22

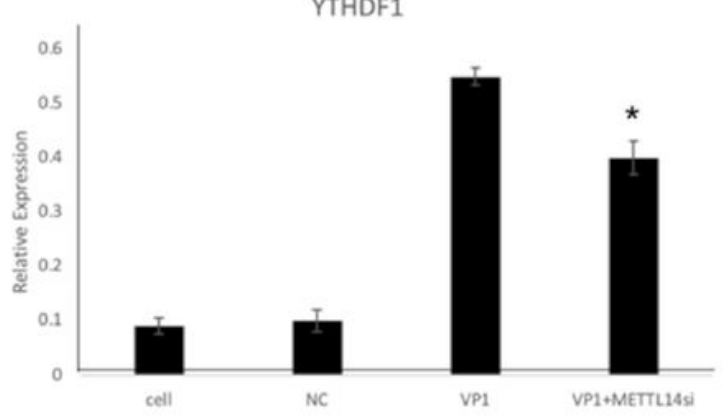

cell NC VP1 VP1+YTHDF1si

GAPDH

cell NC VP1 VP1+YTHDF1si
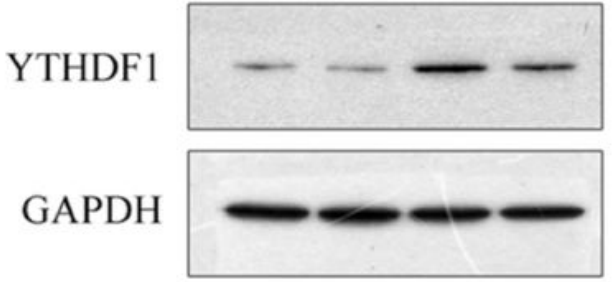

YTHDF1

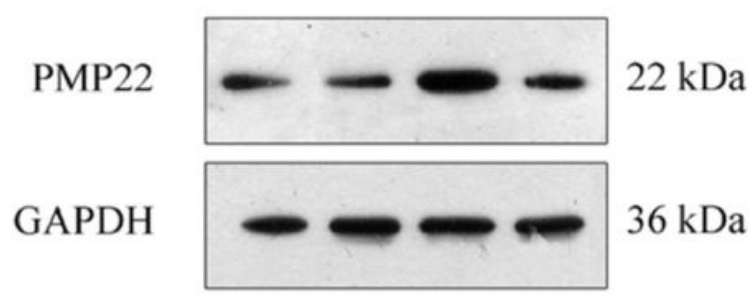

PMP22

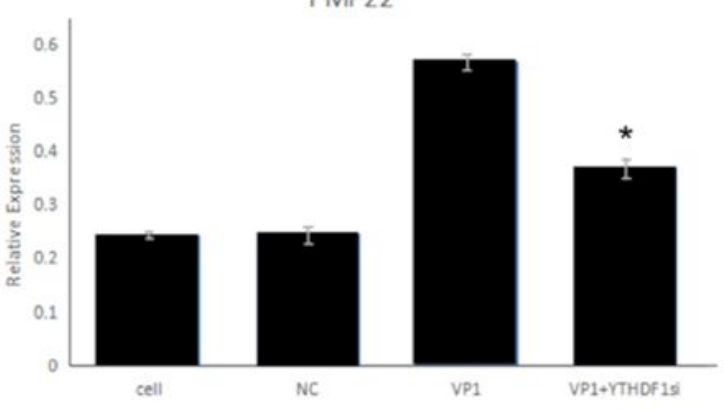

Figure 4

Western blot analysis for EV71-VP1 affected the targeting of m6A methylation and PMP22. A: Gene sequence identifies the position of siRNA design; B: Western blotting showed that the expression of METL14 and YTHDF1 were significantly increased, and the SC after knocking down METL14 and YTHDF1 by siRNA significantly decreased the expression of METL14 and YTHDF1 under the action of VP1 (both $p<0.001$ ); C: Western blotting gray analysis showed that the expression gray levels of siMETTL14 and siYTHDF1 were significantly down-regulated (both $p<0.001$ ); $\mathrm{D}$ : Western blotting showed that the protein expression of 
PMP22 with METL14si and YTHDF1si were significantly decreased; E: Western blotting gray analysis showed that the RNA expression gray levels of PMP22 with METTL14si and YTHDF1si were significantly down-regulated to VP1-cell (respectively $\mathbb{p}=0.006$ and $p=0.011$ ). PMP22, peripheral myelin protein 22; si, silenced by siRNA; NC, negative control; VP1, structural viral protein 1; METTL14『methyltransferase-like protein 14; YTHDF1هYTH N(6)-Methyladenosine RNA Binding Protein 1.

\section{Supplementary Files}

This is a list of supplementary files associated with this preprint. Click to download.

- SupplementaryTableS1.docx

- SupplementaryTableS2.docx

- SupplementaryTableS3.docx 